\title{
EFICÁCIA DE HERBICIDAS NO CONTROLE, EM PÓS-EMERGÊNCIA, DE PLANTAS DANINHAS NA CULTURA DO MILHO
}

\author{
Fernando Tadeu de Carvalho ${ }^{1}$, Maximilian Peruchi ${ }^{2}$ e \\ Rodrigo Rodrigues Bernardes Palazzo ${ }^{2}$
}

${ }^{1}$ Engo ${ }^{\circ}$ Agrônomo., Dr., Professor. FEIS/UNESP, Av. Brasil, 56. Caixa Postal 31, Ilha Solteira, SP 15385-000 ftadeu@bio.feis.unesp.br ${ }^{2}$ Graduando de Agronomia. FEIS/UNESP.

\begin{abstract}
RESUMO
O objetivo do trabalho foi avaliar a eficácia agronômica de herbicidas aplicados no controle em pós-emergência de plantas daninhas e avaliar a seletividade à cultura do milho. O experimento foi desenvolvido no período de dezembro/00 a abril/01, na FEP-UNESP, em Selvíria, MS. O experimento foi conduzido em cultivo convencional e o híbrido de milho utilizado foi o 'Master'. O delineamento experimental adotado foi o de blocos ao acaso, com 9 tratamentos e 4 repetições. Os tratamentos avaliados foram: foramsulfuron (35,0 e 45,5 g/ha), foramsulfuron/iodosulfuron-methyl-sodium (36,0/2,4 e 45,0+3,0

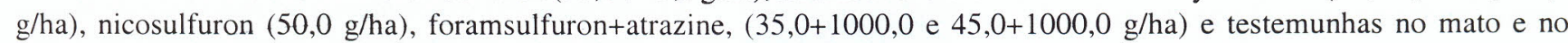
limpo. As aplicações foram realizadas com um pulverizador costal a pressão constante $\left(\mathrm{CO}_{2}\right)$ de $3,2 \mathrm{~kg} / \mathrm{cm}^{2}, \mathrm{com}$ barra equipada com quatro bicos do tipo leque, Teejet $110.03 \mathrm{XR}$, espaçados de $0,5 \mathrm{~m}$ e consumo de calda de $250 \mathrm{l} / \mathrm{ha}$. Os tratamentos herbicidas foram seletivos à cultura de milho. Os tratamentos foramsulfuron (45,5 g/ha), foramsulfuron/ iodosulfuron-methyl-sodium $(36,0 / 2,4$ e 45,0/3,0 g/ha) e foramsulfuron+atrazine $(45,0+1000,0 \mathrm{~g} / \mathrm{ha})$ foram eficazes no controle de Bidens pilosa, Commelina benghalensis e Digitaria horizontalis. Os tratamentos foramsulfuron ( $35,0 \mathrm{~g} / \mathrm{ha})$, nicosulfuron $(50,0 \mathrm{~g} / \mathrm{ha})$ e foramsulfuron+atrazine $(35,0+1000,0 \mathrm{~g} / \mathrm{ha})$ foram eficazes no controle de $B$. pilosa e C. benghalensis. Não foi observado efeito fitotóxico dos herbicidas ao milho.
\end{abstract}

Palavras-chave: infestantes, interferência, Zea mays.

\section{ABSTRACT \\ Efficacy of herbicides in postemergence control of weeds in corn crop}

The objective of this research was to evaluate the agronomic efficiency of herbicides applied as a postemergence treatment on the control of weeds and to evaluate the tolerance of the treatments for corn. The experiment was developed during the period from december/00 to april/01, at FEP-UNESP at Selvíria, Mato Grosso do Sul, Brasil. The experiment was carried out in a conventional system and the hybrid corn used was 'Master' at a spacing of 0.9 m between rows with 5.4 seeds per meter. The experimental design was a randomized complete block, with 9 treatments and 4 replicates with 5 lines of the crop. Plot size was $4.5 \times 5.0 \mathrm{~m}$. The treatments were: foramsulfuron ( 35.0 and $45.5 \mathrm{~g} / \mathrm{ha}$ ), foramsulfuron/iodosulfuronmethyl-sodium $(36.0 / 2.4$ and $45.0 / 3.0 \mathrm{~g} / \mathrm{ha})$, nicosulfuron $(50.0 \mathrm{~g} / \mathrm{ha})$, foramsulfuron+atrazine $(35.0+1000.0$ and $45.0+1000.0$ $\mathrm{g} / \mathrm{ha}$ ) and a weed-free and a weeded control. The applications were accomplished with a manual sprayer and constant pressure $\left(\mathrm{CO}_{2}\right)$ of $45 \mathrm{lb} / \mathrm{pol}^{2}$, equipped with four Teejet $110.03 \mathrm{XR}$ nozzles, spaced at $0.5 \mathrm{~m}$ and volume of $250 \mathrm{l} / \mathrm{ha}$. The treatments were selective for the crop. The treatments foramsulfuron $(45.5 \mathrm{~g} / \mathrm{ha})$, foramsulfuron/iodosulfuron-methyl-sodium (36.0/2.4 and 45.0/3.0 g/ha) and foramsulfuron+atrazine $(45.0+1000.0 \mathrm{~g} / \mathrm{ha})$ were efficient in the control of Bidens 
Fernando Tadeu de Carvalho et al.

pilosa, Commelina benghalensis and Digitaria horizontalis. The treatments foramsulfuron (35.0 g/ha), nicosulfuron (50.0 g/ ha) and foramsulfuron+atrazine $(35.0+1000.0 \mathrm{~g} / \mathrm{ha})$ were efficient in the control of $B$. pilosa and $C$. benghalensis.

Key words: interference, weeds, Zea mays.

\section{INTRODUÇÃO}

As condições edafoclimáticas destacam o Brasil como um país de grande potencial para a cultura do milho. Entretanto, o clima tropical é também muito favorável à ocorrência de uma grande quantidade de plantas daninhas, que interferem no desenvolvimento e na produção da cultura.

Os efeitos negativos detectados no crescimento e produtividade da cultura do milho, devidos à presença de plantas daninhas, já foram observados por vários autores (Carvalho \& Galli, 1993; Velini et al., 1993; Silva et al., 1993; Rossi et al., 1996, Pinto et al., 2000 e Constantin et al., 2000). Segundo dados da ANDEF (1987) as perdas mundiais de produção de grãos de milho devido à ocorrência de plantas daninhas são de $13 \%$ ao ano; no entanto, no clima tropical do Brasil, as perdas podem chegar a $85 \%$ (Blanco, 1985).

Existem diversos métodos para se controlar as plantas daninhas numa cultura, que variam desde os mecânicos e os culturais, até o uso de produtos químicos. No milho, o controle químico tem sido muito utilizado, em função do grande rendimento operacional que se consegue e devido à desvantagem dos métodos mecânicos de não poderem ser utilizados nos períodos mais chuvosos.

O objetivo do trabalho foi avaliar a eficácia agronômica de herbicidas aplicados no controle, em pós-emergência, de plantas daninhas e avaliar a seletividade dos tratamentos para a cultura do milho.

\section{MATERIAL E MÉTODOS}

O experimento foi conduzido no período de dezembro/00 a abril/01, na Fazenda de Ensino e Pesquisa (FEP) da UNESP - FEIS, situada a $20^{\circ} 22^{\prime}$ de latitude sul e $51^{\circ} 22^{\prime}$ de longitude oeste, a 335 metros de altitude e localizada no município de Selvíria, MS, enquadrada em região de Cerrado, no sudeste de Mato Grosso do Sul. O solo da área experimental é classificado como Latossolo Vermelho-Escuro textura argilosa, com $44 \%$ de argila, $8 \%$ de silte e $48 \%$ de areia.

Antes da instalação do experimento a área foi preparada convencionalmente com uma aração e duas gradagens niveladoras, sendo a última imediatamente antes da semeadura, de modo a eliminar as plantas daninhas que já haviam germinado.

A semeadura foi realizada em 10/12/00, utilizando-se a semeadora Jumil modelo 2600 . O híbrido de milho utilizado foi o 'Master', com espaçamento de 0,9 m entre-linhas, com 5,4 sementes por metro linear. Após a semeadura ocor- reu uma chuva de $25,9 \mathrm{~mm}$ e a emergência ocorreu em 5 dias.

Os tratos culturais realizados na área experimental foram os normais necessários para a cultura. A adubação foi realizada concomitantemente à semeadura, utilizando-se a fórmula comercial 8-28-16+0,3\% de zinco, à base de 250 $\mathrm{kg} / \mathrm{ha}$, e adubação de cobertura aos 40 dias após a semeadura, com a fórmula $20-0-20$ a $220 \mathrm{~kg} / \mathrm{ha}$.

Foi realizada uma aplicação do inseticida Tracer na dose $40 \mathrm{ml} / \mathrm{ha}$, aos 25 dias após a semeadura, para o controle de lagartas do cartucho. A aplicação foi realizada com um pulverizador de barra tratorizado e volume de calda de $200 \mathrm{l} /$ ha.

As características dos herbicidas utilizados no experimento estão apresentados na Tabela 1. Além dos tratamentos com herbicidas foram utilizadas ainda uma testemunha no mato e outra no limpo. Na testemunha no limpo o controle das plantas daninhas foi feito manualmente, de 15 em 15 dias, até o fechamento da cultura.

O delineamento experimental adotado foi o de blocos ao acaso, com nove tratamentos e quatro repetições. Cada parcela constou de 5 linhas da cultura com $5 \mathrm{~m}$ de comprimento e $4,5 \mathrm{~m}$ de largura, totalizando $22,5 \mathrm{~m}^{2}$, com $20 \mathrm{~m}^{2}$ de área aplicada. A área total do experimento (36 parcelas) foi de $810 \mathrm{~m}^{2}$.

As aplicações dos herbicidas foram realizadas com um pulverizador costal com pressão constante $\left(\mathrm{CO}_{2}\right)$ de $3,2 \mathrm{~kg} /$ $\mathrm{cm}^{2}$, provido de tanque com capacidade de dois litros (garrafas descartáveis) e com barra equipada com quatro bicos do tipo leque, Teejet $110.03 \mathrm{XR}$, espaçados de 0,5 metro. O volume de calda aplicado foi de 250 litros por hectare.

As aplicações foram realizadas em pós-emergência da cultura e das plantas daninhas, aos 20 dias após a semeadura (DAS) no dia 30/12/00, das 20h00 às 20h45. Na ocasião, as plantas daninhas de folhas largas estavam com 2-4 folhas e as gramíneas com 1-2 perfilhos. O solo encontrava-se com umidade, em decorrência de chuvas ocorridas nos dias anteriores. A temperatura ambiente, marcada no início das aplicações foi de $31^{\circ} \mathrm{C}$, a umidade relativa do ar de $68 \%$, e a velocidade do vento inferior a $3 \mathrm{~km} / \mathrm{hora}$.

As avaliações de fitotoxicidade foram realizadas até o desaparecimento dos sintomas e as avaliações de controle de plantas daninhas até o fechamento da cultura.

A eficiência dos herbicidas no controle das plantas daninhas foi avaliada aos 7,21 e 35 dias após a aplicação (DAA) dos tratamentos. Utilizou-se uma escala visual onde $0 \%=$ nenhum controle e $100 \%=$ controle total das plantas daninhas. Considerou-se como eficiente o controle superior a $80 \%$. 
Eficácia de herbicidas na cultura do milho

Tabela 1. Características dos herbicidas utilizados no experimento. FEP, Selvíria, MS. 2000/2001.

\begin{tabular}{|c|c|c|c|c|c|}
\hline \multicolumn{2}{|c|}{ Herbicida } & \multicolumn{2}{|c|}{ Dose } & \multirow{3}{*}{ Concentração } & \multirow{3}{*}{ Formulação } \\
\hline \multirow{2}{*}{ Nome técnico } & \multirow{2}{*}{ Nome comercial } & I.A. & P.C. & & \\
\hline & & (g/ha) & (g ou $1 / h a)$ & & \\
\hline Foramsulfuron & Tribute & 35,0 & $100 \mathrm{~g}$ & $350 \mathrm{~g} / \mathrm{kg}$ & $\mathrm{GRDA}^{2}$ \\
\hline Foramsulfuron & Tribute & 45,5 & $130 \mathrm{~g}$ & $350 \mathrm{~g} / \mathrm{kg}$ & GRDA \\
\hline Foramsulfuron/Iodosulfuron ${ }^{1}$ & Equip Plus & $36,0 / 2,4$ & $120 \mathrm{~g}$ & $320 \mathrm{~g} / \mathrm{kg}$ & GRDA \\
\hline Foramsulfuron/Iodosulfuron ${ }^{1}$ & Equip Plus & $45,0 / 3,0$ & $150 \mathrm{~g}$ & $320 \mathrm{~g} / \mathrm{kg}$ & GRDA \\
\hline Nicosulfuron & Sanson & 50,0 & 1,251 & $40 \mathrm{~g} / 1$ & $\mathrm{SC}^{3}$ \\
\hline Foramsulfuron+atrazine & Tribute+Atrazinax & $35,0+1000,0$ & $100 \mathrm{~g}+2,01$ & $350 \mathrm{~g} / \mathrm{kg}+500 \mathrm{~g} / 1$ & GRDA+SC \\
\hline Foramsulfuron+atrazine & Tribute+Atrazinax & $45,5+1000,0$ & $130 \mathrm{~g}+2,01$ & $350 \mathrm{~g} / \mathrm{kg}+500 \mathrm{~g} / \mathrm{l}$ & $\mathrm{GRDA}+\mathrm{SC}$ \\
\hline
\end{tabular}

${ }^{1}$ Iodosulfuron-methyl-sodium

${ }^{2}$ Grânulos dispersíveis em água

${ }^{3}$ Suspensão concentrada

As avaliações de seletividade foram realizadas aos 7 , 14 e 21 dias após a aplicação (DAA) dos tratamentos. Para se determinar o efeito fitotóxico dos herbicidas utilizou-se uma escala de percentagem de injúria, onde $0 \%=$ nenhuma fitotoxicidade e $100 \%$ = destruição total das plantas da cultura.

A avaliação de produtividade foi realizada aos 118 dias após a semeadura, no dia 07/04/01, coletando-se os grãos da área útil, ou seja, nos $2 \mathrm{~m}^{2}$ centrais de cada parcela.

\section{RESULTADOS E DISCUSSÃO}

As espécies daninhas que ocorreram com maior freqüência no experimento (Tabela 2) foram o picão-preto (Bidens pilosa), a trapoeraba (Commelina benghalensis) e o capim-colchão (Digitaria horizontalis). Os dados de controle das plantas daninhas, estão contidos na Tabela 3.

Bidens pilosa, Commelina benghalensis e Digitaria horizontalis foram relacionadas por Forster (1991) entre as principais espécies daninhas de áreas de cultivo de milho. $C$. benghalensis tem se destacado em lavouras de milho pela sua frequência e por ser considerada planta de difícil controle (Marochi, 2000). Na cultura do milho, em sistema convencional, a trapoeraba foi recentemente relatada por Foloni et al. (2000).

Observa-se, pelos dados de controle das plantas daninhas, que o tratamento foramsulfuron $(45,5 \mathrm{~g} / \mathrm{ha})$ foi eficien- te no controle de B. pilosa, $C$. benghalensis e $D$. horizontalis, proporcionando níveis médios de controle de 96, 93 e 80\%, respectivamente, aos 35 DAA. Na dose de $35,0 \mathrm{~g} / \mathrm{ha}$, o herbicida foi eficiente no controle de B. pilosa e $C$. benghalensis, proporcionando níveis médios de controle de $95 \%$ e $88 \%$, respectivamente, aos 35 DAA. Para a espécie $D$. horizontalis, a dose de $35,0 \mathrm{~g} / \mathrm{ha}$ de foramsulfuron proporcionou níveis médios de controle abaixo de $80 \%$.

O herbicida foramsulfuron/iodosulfuron $(36,0+2,4$ e $45,0+3,0 \mathrm{~g} / \mathrm{ha}$ ) foi eficiente no controle das plantas daninhas, proporcionando níveis médios de controle de $100 \%$ para $B$. pilosa, acima de $90 \%$ para C. benghalensis e de $80 \%$ para $D$. horizontalis, aos 35 DAA.

O herbicida nicosulfuron $(50 \mathrm{~g} / \mathrm{ha})$ foi eficiente no controle de $B$. pilosa e $C$. benghalensis, proporcionando níveis médios de controle de 92 e $88 \%$, respectivamente, aos 35 DAA. Para a espécie $D$. horizontalis, o tratamento proporcionou níveis médios de controle abaixo de $80 \%$. Esses resultados estão de acordo com Lorenzi (2000) apenas para a espécie $B$. pilosa.

O tratamento foramsulfuron+atrazine $(45,5+1000,0 \mathrm{~g} /$ ha) foi eficiente no controle de B. pilosa, C. benghalensis e $D$. horizontalis, proporcionando níveis médios de controle de 100,97 e $84 \%$, respectivamente, aos 35 DAA. Na dose de $35,0+1000,0 \mathrm{~g} / \mathrm{ha}$, o herbicida foi eficiente no controle de B. pilosa e $C$. benghalensis, proporcionando níveis médios de controle de 97 e 92\%, respectivamente, aos 35 DAA. Para a espécie $D$. horizontalis, a dose de $35,0+1000 \mathrm{~g} / \mathrm{ha}$ do

Tabela 2. Espécies daninhas que ocorreram com maior frequiência no experimento. FEP, Selvíria, MS. 2000-2001.

\begin{tabular}{lcccc}
\hline \multirow{2}{*}{ Espécie Daninha } & \multicolumn{3}{c}{ Número médio de plantas $/ \mathbf{m}^{2}$} \\
\cline { 2 - 5 } & 7 DAA & 14 DAA & 21 DAA & 35 DAA \\
\hline Bidens pilosa (picão-preto) & 35 & 33 & 32 & 35 \\
Commelina benghalensis (trapoeraba) & 9 & 13 & 15 & 15 \\
Digitaria horizontalis (capim-colchão) & 28 & 30 & 97 & 50 \\
\hline Total & 72 & 76 & & 100 \\
\hline
\end{tabular}

DAA= dia após a aplicação. 
Fernando Tadeu de Carvalho et al.

Tabela 3. Controle de plantas daninhas, obtido com herbicidas de pós-emergência na cultura do milho. FEP, Selvíria, MS. 2000-2001.

\begin{tabular}{|c|c|c|c|c|c|c|c|c|c|c|}
\hline \multirow{4}{*}{ Tratamento } & \multirow{4}{*}{$\begin{array}{c}\text { Dose } \\
\text { (g/ha) }\end{array}$} & \multicolumn{9}{|c|}{ Controle $(\%)$} \\
\hline & & \multicolumn{3}{|c|}{ B. pilosa } & \multicolumn{3}{|c|}{ C. benghalensis } & \multicolumn{3}{|c|}{ D. horizontalis } \\
\hline & & \multicolumn{3}{|c|}{$\mathrm{DAA}^{2}$} & \multicolumn{3}{|c|}{ DAA } & \multicolumn{3}{|c|}{ DAA } \\
\hline & & 7 & 21 & 35 & 7 & 21 & 35 & 7 & 21 & 35 \\
\hline Testemunha no mato & -- & 0 & 0 & 0 & 0 & 0 & 0 & 0 & 0 & 0 \\
\hline Testemunha no limpo & -- & 100 & 100 & 100 & 100 & 100 & 100 & 100 & 100 & 100 \\
\hline Foramsulfuron & 35,0 & 41 & 89 & 95 & 24 & 46 & 88 & 35 & 55 & 74 \\
\hline Foramsulfuron & 45,5 & 48 & 89 & 96 & 28 & 51 & 93 & 39 & 65 & 80 \\
\hline Foramsulfuron/iodosulfuron ${ }^{1}$ & $36,0 / 2,4$ & 70 & 98 & 100 & 24 & 51 & 90 & 29 & 66 & 80 \\
\hline Foramsulfuron/iodosulfuron ${ }^{1}$ & $45,0 / 3,0$ & 76 & 98 & 100 & 26 & 60 & 92 & 32 & 70 & 80 \\
\hline Nicosulfuron & 50,0 & 46 & 78 & 92 & 30 & 51 & 88 & 42 & 71 & 79 \\
\hline Foramsulfuron+atrazine ${ }^{1}$ & $35,0+1000,0$ & 91 & 97 & 97 & 87 & 87 & 92 & 40 & 68 & 72 \\
\hline Foramsulfuron+atrazine $^{1}$ & $45,5+1000,0$ & 98 & 100 & 100 & 90 & 90 & 97 & 45 & 71 & 84 \\
\hline
\end{tabular}

'Foi adicionada à calda de pulverização o espalhante adesivo Hoefix a $0,25 \%$ v/v

${ }^{2} \mathrm{DAA}=$ dias após a aplicação

Tabela 4. Fitotoxicidade dos herbicidas e produção de grãos de milho no experimento. FEP, Selvíria, MS. 2000.

\begin{tabular}{|c|c|c|c|c|c|}
\hline \multirow{2}{*}{ Tratamento } & \multirow{2}{*}{$\begin{array}{c}\text { Dose } \\
\text { (g/ha) }\end{array}$} & \multicolumn{3}{|c|}{ Fitotoxicidade (\%) } & \multirow{2}{*}{$\begin{array}{c}\text { Produção } \\
\text { (kg/ha) }\end{array}$} \\
\hline & & $7 \mathbf{D A A}^{1}$ & $14 \mathrm{DAA}^{1}$ & $21 \mathrm{DAA}^{1}$ & \\
\hline Testemunha no mato & -- & 0,0 & 0,0 & 0,0 & $2479 b^{2}$ \\
\hline Testemunha no limpo & -- & 0,0 & 0,0 & 0,0 & 5490 a \\
\hline Foramsulfuron & 35,0 & 4,8 & 0,5 & 0,0 & 5155 a \\
\hline Foramsulfuron & 45,5 & 5,8 & 1,0 & 0,0 & 5172 a \\
\hline Foramsulfuron/iodosulfuron & $36,0 / 2,4$ & 6,5 & 1,5 & 0,0 & 4578 a \\
\hline Foramsulfuron/iodosulfuron & $45,0 / 3,0$ & 8,5 & 3,0 & 0,0 & 4687 a \\
\hline Nicosulfuron & 50,0 & 6,3 & 1,5 & 0,0 & $4023 \mathrm{ab}$ \\
\hline Foramsulfuron+atrazine & $35,0+1000$ & 5,8 & 1,0 & 0,0 & 4681 a \\
\hline Foramsulfuron+atrazine & $45,5+1000$ & 9,3 & 3,0 & 0,0 & 5272 a \\
\hline Média Geral & & & & & 4615 \\
\hline Teste f (tratamentos) & & & & & $6,07 * *$ \\
\hline Coeficiente de Variação (\%) & & & & & 16,1 \\
\hline $\operatorname{DMS}(5 \%)$ & & & & & 1790 \\
\hline
\end{tabular}

'DAA = dias após a aplicação

${ }^{2}$ Médias seguidas de letras iguais nas colunas, não diferem entre si ao nível de significância de 5\%, pelo teste de Tukey.

tratamento, proporcionou níveis médios de controle abaixo de $80 \%$.

Os dados médios de fitotoxicidade dos herbicidas e de produtividade da cultura estão apresentados na Tabela 4. Os tratamentos herbicidas provocaram sintomas de fitotoxicidade inicial, caracterizados por um leve amarelecimento das folhas da cultura. Nas avaliações subsequentes os sintomas foram desaparecendo gradativamente e, aos 21 DAA, não foram mais constatados nenhum sintoma de fitotoxicidade.

Os dados de produtividade confirmam os efeitos prejudiciais da competição entre cultura e plantas daninhas, já constatado por outros autores (Carvalho \& Galli, 1993; Velini et al., 1993 e Silva et al., 1993). A convivência com as plan- tas daninhas prejudicou significativamente a produtividade da cultura no tratamento testemunha no mato. Observou-se que a queda de produtividade da testemunha no mato em relação aos tratamentos com controle foi de $49 \%$.

\section{CONCLUSÕES}

Os herbicidas foramsulfuron $(35,0$ e $45,5 \mathrm{~g} / \mathrm{ha})$, foramsulfuron+iodosulfuron-methyl-sodium $(36,0 / 2,4$ e 45,0/ $3,0 \mathrm{~g} / \mathrm{ha})$, nicosulfuron $(50,0 \mathrm{~g} / \mathrm{ha})$, foramsulfuron+atrazine $(35,0+1000,0$ e 45,0+1000,0 g/ha) são seletivos às plantas de milho cultivar 'Master'. 
Os herbicidas foramsulfuron $(45,5 \mathrm{~g} / \mathrm{ha})$, foramsulfuron+iodosulfuron-methyl-sodium (36,0/2,4 e 45,0/ $3,0 \mathrm{~g} / \mathrm{ha})$ e foramsulfuron+atrazine $(45,0+1000,0 \mathrm{~g} / \mathrm{ha})$ são eficientes no controle de Bidens pilosa, Commelina benghalensis e Digitaria horizontalis.

Os herbicidas foramsulfuron $(35,0 \mathrm{~g} / \mathrm{ha})$, nicosulfuron $(50,0 \mathrm{~g} / \mathrm{ha})$ e foramsulfuron+atrazine $(35,0+1000,0 \mathrm{~g} / \mathrm{ha})$ são eficientes no controle de $B$. pilosa e $C$. benghalensis.

\section{LITERATURA CITADA}

ASSOCIAÇÃO NACIONAL DE DEFESA VEGETAL ANDEF. Defesa vegetal. São Paulo, SP: ANDEF, 1987. $19 \mathrm{p}$.

BLANCO, H.G. Ecologia das plantas daninhas - competição de plantas daninhas em culturas brasileiras. In: Controle integrado de plantas daninhas. 2 ed. São Paulo: CREA, 1985. p. 43-75.

CARVALHO, F.T.; GALLI, A.J.B. Nova formulação de alachlor na seletividade e no controle de plantas daninhas na cultura do milho. In: CONGRESSO BRASILEIRO DE HERBICIDAS E PLANTAS DANINHAS, 19. Londrina, PR, 1993. Resumos... Londrina: SBHED, 1993. p. 131.

CONSTANTIN, J.; OLIVEIRA JR., R.S.; MACIEL, C.D.G.; ROSSI, J.M. Utilização de isoxaflutole e isoxaflutole+atrazina para o manejo de plantas daninhas em milho. In: CONGRESSO BRASILEIRO DA CIÊNCIA DAS PLANTAS DANINHAS, 22. Foz do Iguaçu, PR, 2000. Resumos... Londrina: SBCPD, 2000. p. 280.

FOLONI, L.L.; CORBO, E; RODRIGUES, J.D.; ONO, E.O. Tolerância e eficiência do glyphosate aplicado em pósemergência em milho transgênico em plantio direto. In: CONGRESSO BRASILEIRO DA CIÊNCIA DAS PLANTAS DANINHAS, 22. Foz do Iguaçu, PR, 2000. Resumos... Londrina: SBCPD, 2000. p. 283.
FORSTER, R. Controle das plantas invasoras na cultura do milho. Série técnica, no 5. Campinas, SP: Fundação Cargill, 1991. 89p.

LORENZI, H. Manual de identificação e controle de plantas daninhas: plantio direto e convencional. 5 ed. Nova Odessa, SP: Plantarum, 2000. 339 p.

MAROCHI, A.I. Eficácia de glyphosate isolado ou em mistura no controle de Commelina benghalensis em aplicação de pré-plantio com complementação de glyphosate 30 dias após emergência da soja Roundup Ready. In: CONGRESSO BRASILEIRO DA CIÊNCIA DAS PLANTAS DANINHAS, 22. Foz do Iguaçu, PR, 2000. Resumos... Londrina: SBCPD, 2000. p. 174.

PINTO, J.J.O.; SPERANDIO, C.A.; FERREIRA, F.B.; LAMEGO, F.P. Controle de plantas daninhas na cultura do milho com herbicidas de aplicação em pré e pósemergência. In: CONGRESSO BRASILEIRO DA CIENCIA DAS PLANTAS DANINHAS. Foz do Iguaçu, PR, 2000. Resumos... Londrina: SBCPD, 2000. p. 279.

ROSSI, I. H.; OSUNA, J. A.; ALVES, P. L. C. A.; BEZUTT, A. J. Interferência das plantas daninhas sobre algumas características agronômicas e a produtividade de sete cultivares de milho. Planta Daninha, Botucatu, v.14, n.2, p. 134-148, 1996.

SILVA, M. S. J.; ASMUS, G. L.; CORREA, A. M. Estudo do herbicida nicosulfuron no controle de plantas daninhas na cultura do milho (Zea mays L.). In: CONGRESSO BRASILEIRO DE HERBICIDAS E PLANTAS DANINHAS, 19. Londrina, PR, 1993. Resumos... Londrina: SBHED, 1993. p. 148-149.

VELINI, E. D.; FREDERICO, L. A.; BICUDO, S. J.; ANTUNIASSI, U. R. Eficiência de herbicidas pré e pós emergentes iniciais recomendados para a cultura do milho, no controle de plantas daninhas e avaliação de efeitos dos mesmos sobre a produtividade da cultura. In: CONGRESSO BRASILEIRO DE HERBICIDAS E PLANTAS DANINHAS, 19. Londrina, PR, 1993. Resumos... Londrina: SBHED, 1993. p. 141-142. 
